\title{
Supramolecular hybrid structures and gels from host-guest interactions between $\alpha$ - cyclodextrin and PEGylated organosilica nanoparticles
}

Article

Accepted Version

Serres-Gómez, M., González-Gaitano, G., Kaldybekov, D. B., Mansfield, E. D. H., Khutoryanskiy, V. V., Isasi, J. R. and Dreiss, C. A. (2018) Supramolecular hybrid structures and gels from host-guest interactions between $\alpha$-cyclodextrin and PEGylated organosilica nanoparticles. Langmuir, 34 (36). pp. 10591-10602. ISSN 0743-7463 doi: https://doi.org/10.1021/acs.langmuir.8b01744 Available at https://centaur.reading.ac.uk/79045/

It is advisable to refer to the publisher's version if you intend to cite from the work. See Guidance on citing.

To link to this article DOI: http://dx.doi.org/10.1021/acs.langmuir.8b01744

Publisher: American Chemical Society

All outputs in CentAUR are protected by Intellectual Property Rights law, including copyright law. Copyright and IPR is retained by the creators or other copyright holders. Terms and conditions for use of this material are defined in the End User Agreement. 


\section{www.reading.ac.uk/centaur}

\section{CentAUR}

Central Archive at the University of Reading

Reading's research outputs online 


\title{
Supramolecular hybrid structures and gels from host-guest interactions between $\alpha$ - cyclodextrin and PEGylated organosilica nanoparticles
}

Mariana Serres-Gómez ${ }^{1,4}$, Gustavo González-Gaitano ${ }^{1}$, Daulet B. Kaldybekov ${ }^{2,3}$, Edward D.H. Mansfield ${ }^{2}$, Vitaliy V. Khutoryanskiy ${ }^{2}$, José Ramón Isasi ${ }^{1 *}$, and Cécile A. Dreiss ${ }^{4 *}$

${ }^{1}$ Department of Chemistry. University of Navarra. 31080 Pamplona, Spain

${ }^{2}$ Reading School of Pharmacy, University of Reading, Whiteknights, PO box 224, RG6 6AD Reading, $U K$

${ }^{3}$ Faculty of Chemistry and Chemical Technology, Al-Farabi Kazakh National University, Almaty 050040, Kazakhstan

${ }^{4}$ Institute of Pharmaceutical Science, School of Cancer \& Pharmaceutical Sciences, Franklin-Wilkins Building, 150 Stamford Street, SE1 9NH London, King's College London, $U K$

\section{Corresponding Authors}

*E-mail: cecile.dreiss@kcl.ac.uk

*E-mail: jrisasi@unav.es

\begin{abstract}
Polypseudorotaxanes are polymer chains threaded by molecular rings that are free to unthread; these 'pearl-necklace' can self-assemble further, leading to higher order supramolecular structures with interesting functionalities. In this work, the complexation between $\alpha$-cyclodextrin ( $\alpha$-CD), a cyclic oligosaccharide of glucopyranose units, and polyethylene glycol (PEG) grafted to silica nanoparticles was studied. The threading of $\alpha$-CD onto the polymeric chains leads to their aggregation into bundles, followed by either the precipitation of the inclusion complex, or the formation of a gel phase, in which silica nanoparticles are incorporated.

The kinetics of threading, followed by turbidimetry, revealed a dependence of the rate of complexation on the following parameters: the concentration of $\alpha-\mathrm{CD}$, temperature, PEG length $\left(750,4,000\right.$ and 5,000 $\left.\mathrm{g} \cdot \mathrm{mol}^{-1}\right)$, whether the polymer is grafted or free in solution,
\end{abstract}


and the density of grafting. Complexation is slower, and temperature has a higher impact on PEG grafted on silica nanoparticles compared to PEG free in solution. Thermodynamic parameters extracted from the transition state theory showed that inclusion complex formation is favoured with grafted PEG compared to free PEG and establish a ratio of complexation of 5-6 EO units per cyclodextrin. The complexation yields, determined by gravimetry, revealed that much higher yields are obtained with longer chains and higher grafting density. TGA analysis and FTIR spectroscopy on the inclusion complex corroborate the number of macrocycles threaded on the chains. A solgel transition was observed with the longer PEG chain $(5 \mathrm{k})$ at specific mixing ratios; oscillatory shear rheology measurements confirmed a highly solid-like behaviour, with an elastic modulus $G^{\prime}$ of up to $25 \mathrm{kPa}$, higher than in the absence of silica. These results thus provide the key parameters dictating inclusion complex formation between cyclodextrin and PEG covalently attached to colloidal silica, and demonstrate a facile route towards soft nanoparticle gels based on host-guest interactions.

\section{KEYWORDS}

Polypseudorotaxanes; PEG; cyclodextrins; nanocomposite gels; silica nanoparticles; threading kinetics.

\section{INTRODUCTION}

Cyclodextrins (CDs), cyclic oligosaccharides consisting of six or more glucopyranose units obtained from the enzymatic degradation of starch, ${ }^{1}$ form inclusion complexes with water-soluble polymer chains through non-covalent interactions, as first reported by Akira Harada ${ }^{2}$. When poly(ethylene glycol) (PEG) and $\alpha-\mathrm{CD}$ aqueous solutions are mixed, a complex is spontaneously formed by the threading of $\alpha$-CD molecules onto the polymer chain ${ }^{3,4}$. The driving forces include hydrophobic and van der Waals interactions between the cavity of $\alpha-\mathrm{CD}$ and the $-\mathrm{CH}_{2} \mathrm{CH}_{2} \mathrm{O}$ - units of PEG, in addition to hydrogen bonding between the hydroxyl groups of adjacent macrocycles threaded onto the polymer chain ${ }^{4,5}$. In these pearl necklace structures, referred to as pseudopolyrotaxanes (PPR), a maximum of two ethylene glycol (EO) units are included within each $\alpha$-CD cavity ${ }^{3,6}$. The kinetics of the complexation depends on temperature, solvent composition and the molecular weight of the polymer ${ }^{7}$. For instance, low temperatures and highly structured 
solvents (with a strong hydrogen bonding capacity) favour the threading process ${ }^{7,8}$. In addition, the threaded $\alpha-\mathrm{CD}$ rings on adjacent polymer chains aggregate due to hydrophobic interactions, causing an increase in solution turbidity, ultimately leading to precipitation $^{3,4,8,9}$.

At appropriate compositions, these aggregates can act as physical cross-links and induce the formation of self-supported gels ${ }^{6,7}$. Host-guest interactions, as a route towards hierarchical, functional structures, have received increasing attention in the past decade 10,11. A myriad of versatile soft materials have been reported based on the chemical modification of polymers and nanoparticles with suitable macrocycles and complementary guest molecules, for applications in drug delivery and tissue engineering, ${ }^{12-14}$, some undergoing clinical trials ${ }^{15}$. Many of these supramolecular constructs however require expert chemistry and involved synthetic process; there is therefore a drive for simpler routes, using minimal chemistry, in particular by exploiting the spontaneous threading of cyclodextrins on polymer chains as the basis for organising matter. This approach has already produced many functional biomaterials, either for injectable drugdelivery matrices or as self-healing scaffolds for tissue engineering 4,6,11,16-19, some showing promising results in the clinic, for instance polyrotaxanes of hydroxypropyl- $\beta$ cyclodextrins for the treatment of Niemann-Pick C disease ${ }^{20}$.

In this context, using the pearl-necklace structure as a starting point, our objective was to improve functionality by incorporating nanoparticles within the supramolecular aggregates or self-supported gels. Soft nanocomposite hydrogels, the combination of polymer gel matrices with nanoparticles, have generated intense research in the biomedical materials field ${ }^{21,22}$. The 'softness' and hydrated environment characteristic of hydrogels, which resemble natural tissues, can be modulated by the insertion of nanoparticles in order to improve elasticity, biocompatibility, mechanical properties, or to provide specific functionality such as diagnostic (e.g. detection through spectroscopic properties) or therapeutic (e.g. antimicrobial properties of metallic nanoparticles). ${ }^{23}$ Interestingly, previous studies have shown that $\alpha-C D$ can form inclusion complexes with PEG chains that are physically adsorbed on the surface of silica nanoparticles ${ }^{5,7}$, leading to either precipitates, or, at appropriate $\mathrm{CD}$ /polymer compositions, self-supported gels. The interaction between PEO chains chemically grafted onto polystyrene latex particles and cyclodextrins has also been studied: small-angle neutron scattering measurements revealed that the threading of $\alpha-C D$ led to an extension of the polymer chains into the 
bulk $^{24}$. The structure of inclusion complexes formed between $\alpha-C D$ and a PEG brush has been recently examined using neutron reflectivity and grazing incidence wide-angle $\mathrm{X}$ ray scattering, examining specifically graft density and $\alpha-C D$ concentration ${ }^{25}$. Even more recently, the formation of pseudopolyrotaxanes between $\alpha-C D$ and PEG was utilized to form hybrid gels of silver nanoparticles stabilised by a random copolymer of PEG ether methacrylate and polyacrylic acid; the constructs showed antimicrobial activity and were proposed as injectable antibacterial materials ${ }^{23}$. The inclusion complex between $\alpha$ cyclodextrin and an oxyethylene non-ionic ligand (thiol-functionalised Igepal) has also been exploited to mediate the self-assembly of gold nanoparticles into hexagonal closepacking ${ }^{26,27}$. In another study, gold nanoparticles modified with PEG have been incorporated in hydrogels formed by inclusion complexes with $\alpha$-cyclodextrins for the release of doxorubicin ${ }^{28}$.

Silica nanoparticles have numerous potential applications in drug delivery and diagnosis due to their non-toxic nature and biodegradability, ease of synthesis and subsequent surface functionalisation with targeting ligands or "stealth" polymers, and the possibility 29,30 of preparing mesoporous systems with high drug loading ${ }^{30}$. Previously, a methodology for the synthesis of thiolated organosilica nanoparticles have been developed by Khutoryanskiy et al, using the self-condensation of 3mercaptopropyltrimethoxysilane in dimethylsulfoxide in the presence of air ${ }^{31,32}$. Due to the presence of thiol groups on their surface, these nanoparticles were demonstrated to be an excellent model of mucoadhesive nanomedicines, ${ }^{31,33}$ and were also a very good substrate for further covalent surface functionalization with water-soluble polymers such as PEG, using thiol-maleimide click reactions ${ }^{31,33-36}$.

In this work, we exploit the threading of $\alpha-C D$ on these PEGylated organosilica nanoparticles (sub $100 \mathrm{~nm}$ ), where the PEG chains are covalently grafted on the surface of the nanoparticles ${ }^{34}$, and therefore cannot be displaced through complexation. The kinetics of the aggregation process and the complexation yields with different nanoparticles (NPs) are investigated and compared with those obtained for free PEG and end-capped PEG, using UV-Vis spectroscopy and gravimetric experiments. Thermodynamic parameters are extracted from the transition state theory, which is used to describe the complexation process; this leads to an estimation of the number of macrocycles threaded on the chains, which is compared to values obtained from thermogravimetric methods. At specific compositions, the bundling of the pearl-necklace 
structures induces the formation of gels, where cyclodextrins act as crosslinks for the network. Oscillatory shear measurements reveal a remarkable solid-like behaviour, reminiscent of chemically cross-linked gels. These hybrid gels, to our knowledge, are the first report of soft nanocomposites resulting from the aggregation of pseudopolyrotaxanes covalently attached to silica nanoparticles. 


\section{MATERIALS AND METHODS}

\section{Materials}

Organosilica nanoparticles were synthesized as reported previously ${ }^{31,33-36}$. Briefly, 0.75 $\mathrm{mL}(0.2 \mathrm{~mol} / \mathrm{L})$ of (3-mercaptopropyl)-trimethoxysilane (MPTS) was mixed with $20 \mathrm{~mL}$ of DMSO and $0.5 \mathrm{~mL}$ of $0.5 \mathrm{~mol} / \mathrm{L} \mathrm{NaOH}$ aqueous solution. The reaction was conducted with air bubbling and allowed to proceed for $24 \mathrm{~h}$ under continuous stirring at room temperature protected from light. Nanoparticles were then purified by dialysis against deionized water in the dark (5 L, 8 changes of dialysis solvent) using a 12-14 kDa molecular weight cut-off dialysis membrane tube (Medicell Membranes Ltd, UK) and were stored in the fridge for further use.

The PEGylation step was achieved by mixing $5 \mathrm{~mL}$ aqueous dispersions of thiolatedsilica nanoparticles (SiThio) with $100 \mathrm{mg}$ of methoxypolyethylene glycol maleimide (mPEGMa) of two molecular weights (750 and $5000 \mathrm{Da})$. The reaction mixture was stirred for $16 \mathrm{~h}$ at room temperature protected from light. The free thiol groups react with the maleimide groups, giving the final nanoparticle covalently modified with low or high molecular weight PEG (SiPEG750 and SiPEG5K, respectively). PEGylated nanoparticles were purified by dialysis in the dark as above and stored in the fridge for further use.

$\alpha$-cyclodextrin ( $\geq 98 \%$ ) was purchased from Sigma-Aldrich or WackerChemie AG and used without further purification. Additional experiments were performed with different free PEG polymers: methoxy(polyethylene glycol) maleimide (mPEGMa5K, $\geq 90 \%$ (NMR), $5000 \mathrm{~g} / \mathrm{mol}$ ) and poly(ethylene glycol) methyl ether (mPEG5K, $5000 \mathrm{~g} / \mathrm{mol}$ ) were purchased from Sigma-Aldrich, and poly(ethylene glycol) (PEG4K, 4000 g/mol) was from Acofarma. Structures are shown in Supporting Information (Figure S1).

The composition of the different nanoparticles (PEG approximate percentages) and the concentrations of the stock solutions used in our experiments are shown in Table 1 (see also Supporting information, Figures S2 and S3). Note that SiPEG750 and SiPEG5K\#1 have a significantly lower weight percentage of PEG covalently attached to the nanoparticles than SiPEG5K from batches \#2 and \#3; however, in number density of PEG chains per NP weight, the densities of these two batches are comparable to that of SiPEG750. 
Table 1.Concentration of SiPEG NPs initial stock solutions, PEG composition of NPs, number densities, surface coverage (no. of chains and mass per surface area of NP)*, sizes, polydispersity indices and $\zeta$-potential values

\begin{tabular}{|c|c|c|c|c|c|}
\hline \multirow[b]{2}{*}{ Solution batch } & \multirow{2}{*}{$\begin{array}{c}\text { SiPEG750 } \\
\# 1\end{array}$} & \multicolumn{3}{|c|}{ SiPEG5K } & \multirow{2}{*}{$\begin{array}{c}\text { SiThio } \\
-\end{array}$} \\
\hline & & \#1 & \#2 & \#3 & \\
\hline Stock soln. ${ }^{a}$ (\% wt.) & 1.33 & 1.37 & 1.00 & 1.10 & - \\
\hline \% PEG/NPb (\% wt.) & $\approx 15$ & $\approx 22$ & 58 & 53 & - \\
\hline PEG/SiThioc (mol/kg) & 0.24 & 0.06 & 0.28 & 0.23 & - \\
\hline PEG Conc. (\% wt.) & $0.30^{a}$ & $0.33^{a}$ & $0.58^{b}$ & $0.58^{b}$ & - \\
\hline Diameter $^{d}(n m)$ & 48.0 & 60.3 & - & 69.0 & 47.0 \\
\hline $\mathrm{PDI}^{\mathrm{d}}$ & 0.158 & 0.234 & - & 0.167 & 0.133 \\
\hline No. of PEG chains $/ \mathrm{nm}^{2}$ & 1.66 & 0.39 & 1.95 & 1.59 & - \\
\hline $\mathrm{mg} P E G / \mathrm{m}^{2}$ & 2.1 & 3.3 & 16.2 & 13.3 & - \\
\hline$\zeta$-Potential (mV) & -37.4 & -38.0 & - & -45.0 & -48.4 \\
\hline etermined by dried mas & & $\begin{array}{l}{ }^{\text {CC} C a l c u l ~} \\
{ }^{d} \text { Deter }\end{array}$ & $\begin{array}{l}\text { ted fror } \\
\text { nined b }\end{array}$ & $\begin{array}{l}\text { CPEG/NP } \\
\text { S. }\end{array}$ & \\
\hline
\end{tabular}

As shown in Table 1, SiThio and SiPEG750 have similar diameters whereas SiPEG5K nanoparticles present a larger diameter, which is consistent with the fact that these nanoparticles are modified with longer polymer chains. Polydispersity is low in these samples, although SiPEG5K\#1 shows a higher value. Regarding the $\zeta$-potential values, the nanoparticles present negative values between $-37 \mathrm{mV}$ and $-49 \mathrm{mV}$, suggesting a fairly good colloidal stability ${ }^{37}$.

\section{Methods}

Kinetic analysis

Kinetic experiments were carried out to study the complexation between $\alpha$-CD and PEG by monitoring the evolution of the turbidity. For this purpose, a stock solution of $\alpha-C D$ $12 \%$ w/w was prepared in $\mathrm{H}_{2} \mathrm{O}$. Samples (total volume of $2 \mathrm{~mL}$ ) for $\mathrm{UV}$ analysis were: $6 \% \alpha$-CD with $0.5 \%$ SiPEG5K\#2, $0.66 \%$ SiPEG750 or $0.33 \%$ w/w mPEGMa5K. In the UV-Vis spectrometer (Lambda 35, Perkin Elmer), a small electronic cell-stirrer was 
placed under a quartz cuvette (set to medium-high speed, blanked with ultrapure water). The measurements were recorded straight after the addition of $1 \mathrm{~mL}$ of each solution to the cuvette. The UV-Vis spectrometer recorded absorbance over time using a wavelength of $400 \mathrm{~nm}$ and scan time intervals of $30 \mathrm{~s}$. The inflection points of the sigmoidal curves were calculated with Origin 8.5.1 software by fitting the curves with a logistic function (Supporting information, Figure S4). Additional experiments were performed with a second set of samples using a UV-Vis spectrometer (Agilent 8453) equipped with magnetic stirring and temperature-controlled. A wavelength of $400 \mathrm{~nm}$, a stirring speed of $1400 \mathrm{rpm}$, time interval of scans of $30 \mathrm{~s}$ and a fixed cell temperature between $10 \pm 0.1$ ${ }^{\circ} \mathrm{C}$ and $40 \pm 0.1^{\circ} \mathrm{C}$ were selected. In the case of low and high temperature experiments, sample solutions were thermostated separately. Stock solutions of $10 \% \alpha-\mathrm{CD}, 12 \% \alpha-$ $\mathrm{CD}, 0.6 \%$ PEG4K, $0.66 \% \mathrm{mPEGMa} 5 \mathrm{~K}, 0.66 \% \mathrm{w} / \mathrm{w} \mathrm{mPEG} 5 \mathrm{~K}$ were prepared in ultrapure water.

\section{Determination of the complexation yield}

Three sets of samples were prepared by mixing stock solutions of 1.3\% SiPEG750, 1.4\% SiPEG5K\#1, 1.4\% SiPEG5K\#3, 0.33\% mPEGMa5K, 0.33\% mPEG5K with varying concentrations of $\alpha-C D$. Microtubes were first weighed then increasing amounts of $\alpha-C D$ added to the microtubes, followed by $1.5 \mathrm{~mL}$ of the NP solutions to obtain the following target concentrations: $2 \%, 3 \%, 4 \%, 4.3 \%, 4.8 \%, 5.7 \%$ and $6.5 \% \alpha-\mathrm{CD} w / \mathrm{w}$ (and $7.4 \%$ w/w for additional samples using SiPEG750). The samples were vortex-mixed for $1 \mathrm{~min}$ and placed on a floating rack in an ultrasonic bath for 10 min at a frequency of $37 \mathrm{kHz}$ (Fisherbrand FB11203or Ultrasons-P, Selecta). The samples were left to equilibrate for 3 days and were then centrifuged for $10 \mathrm{~min}$ at $13000 \mathrm{rpm}$, followed by careful removal of all the supernatant and then freeze-dried. All the lyophilised microtubes were weighed and the weight of the complex formed, $W_{c}$, was determined by the mass difference. The complexation yield was calculated using the following equations ${ }^{5,7}$ :

$$
\begin{aligned}
Y \% & =\frac{W_{c}}{W_{S i P E G}+W_{\alpha C D}} \\
Y \% & =\frac{W_{C}}{W_{P E G}+W_{\alpha C D}}
\end{aligned}
$$

Where $W_{c}, W_{S i P E G}, W_{P E G}$ and $W_{\alpha C D}$ are the weights of the complex obtained, PEGylated organosilica nanoparticles, free PEG and $\alpha-C D$, respectively. Equation [2] was used to 
calculate the yields for the samples prepared with free polymers. The products obtained were kept for further characterization.

\section{Characterization of the nanoparticles and complexes}

Dynamic light scattering (DLS) measurements were carried out using a Malvern Zetasizer (Table 1 and Figure S3 in Supporting Information).

Thiolated nanoparticles (SiThio), PEGylated nanoparticles, $\alpha-\mathrm{CD}$ and some of the SiPEG/ $\alpha-C D$ complexes were analysed by thermogravimetry at a heating rate of 10 ${ }^{\circ} \mathrm{C} /$ min under nitrogen atmosphere (Mettler TGA/SDTA 851e) between 25 and $1000{ }^{\circ} \mathrm{C}$, using platinum crucibles.

Infrared spectra of $\alpha-C D$, mPEGMa5K, SiPEG5K and SiPEG/ $\alpha-C D$ complexes were recorded on a FTIR spectrometer (IRAffinity-1S,Shimadzu) using a diamond Attenuated Total Reflectance (ATR) sampling accessory Golden Gate (Specac). The averaged spectra (32 scans) were recorded between $600 \mathrm{~cm}^{-1}$ and $4000 \mathrm{~cm}^{-1}$.

\section{Preparation of the gels and rheological measurements}

Phase behaviour and gel formation was studied for SiPEG5K/ $\alpha-C D$ mixtures. Solutions of SiPEG5K with concentrations ranging between $0.2 \%$ and $1.4 \%$ were mixed with $\alpha$-CD solutions of $2 \%$ to $7.4 \%(\mathrm{w} / \mathrm{w})$. First, solid $\alpha-\mathrm{CD}$ was weighed in a microtube; this was followed by the addition of SiPEG5K solution and of ultrapure water to complete. The samples were vortex-mixed for $1 \mathrm{~min}$ and placed on a floating rack in an ultrasonic bath for $10 \mathrm{~min}$. The samples were left to react for 7 days.

For the rheological measurements, SiPEG5K solutions $(0.5 \% \mathrm{w} / \mathrm{w})$ or mPEGMa5K $(0.3 \% \mathrm{w} / \mathrm{w})$ were mixed with $\alpha-\mathrm{CD}$ (added as a solid) from $2 \%$ to $12 \%(\mathrm{w} / \mathrm{w})$. The samples were vortex-mixed until $\alpha$-CD was completely dissolved, and they were left to stand for at least 2 days.

Shear oscillatory rheology measurements were performed on the gels of $\alpha-C D$ and SiPEG5K or mPEGMa5K using a strain-controlled rheometer (ARES, TA Instruments) fitted with a plate-plate geometry (25 mm diameter). Dynamic strain sweep tests (AS) were performed at a fixed frequency of $6.28 \mathrm{rad} / \mathrm{s}$ to establish the linear viscoelastic region, followed by dynamic frequency sweep measurements (FS) (strain values varying between $0.02 \%$ to $0.1 \%$ carried out at $25^{\circ} \mathrm{C}$ ). 


\section{RESULTS AND DISCUSSION}

\section{Kinetics of threading and thermodynamic parameters}

The kinetics of complexation between SiPEG NPs or free PEG with $\alpha$-CD was followed by UV-vis spectroscopy (Figure 1). The turbidity of the solution increases when the complex is formed and supramolecular aggregation takes place, which is detected as an increase in absorbance with time due to scattering, producing a sigmoid-shaped curve, as reported elsewhere. ${ }^{8,9}$ The first phase of the complexation, where no increase in absorbance is detected, corresponds to the threading phase, according to the multi-step process described by Lo Nostro and co-workers, which involves: (1) the diffusion of the polymer and cyclodextrins; (2) the initial threading of cyclodextrins; (3) the release of solvating water from the polymer and the cavity of cyclodextrin; (4) the sliding of the cyclodextrin along the polymer backbone and the threading of additional molecules; (5) partial dethreading (once the CDs have complexed the polymer, this step in inhibited because of hydrophobic interactions and neighbouring CDs acting as stoppers; (6) the aggregation of the pseudopolyrotaxanes (PPRs) followed by their precipitation (which is detected by an increase in turbidity).
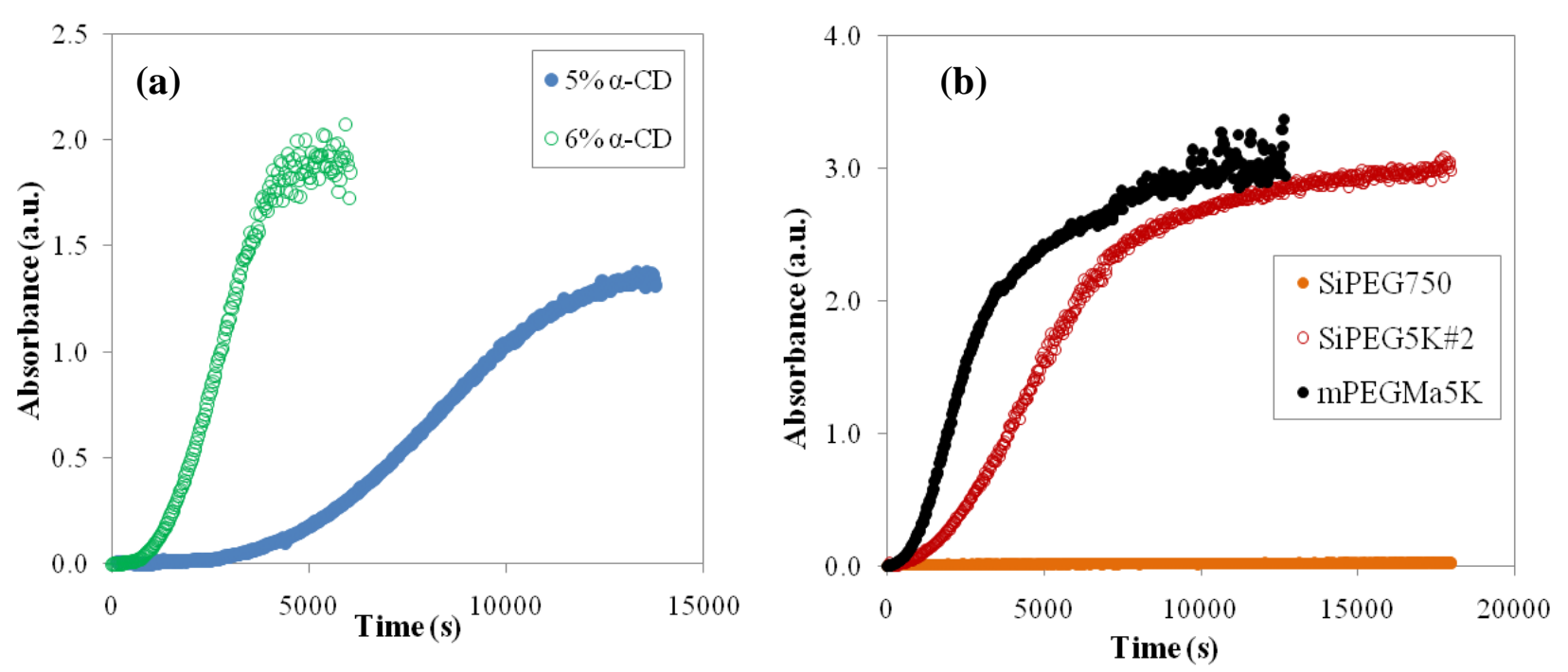

Figure 1. UV-Vis absorbance readings at $400 \mathrm{~nm}$ over time for (a) $5 \%$ and $6 \% \alpha$-CD with $0.55 \%$ SiPEG5K\#3, (b) $6 \% \alpha$-CD with $0.33 \%$ mPEGMa5K, $0.5 \%$ SiPEG5K\#2 (0.3\% PEG equivalent), $0.66 \%$ SiPEG750 (0.15\% PEG equivalent).

The first observation is that complexation between $\alpha-C D$ and PEG does take place even when PEG is grafted onto the nanoparticles (Figure 1b). The kinetics is very clearly dependent on a number of parameters: the concentration of $\alpha$-CD (Figure 1a), whether 
PEG is grafted or not on the silica NPs (mPEGMa5K vs SiPEG5K\#2, Figure 1b), and the length of the grafted PEG (SiPEG750 vs SiPEG5K\#2, Figure 1b). The rate of complexation markedly increases with the concentration of $\alpha-C D$ (Figure 1a): the maximum turbidity is reached three times faster when $\alpha-C D$ concentration is increased from $5 \%$ to $6 \%$. On the other hand, at the highest $\alpha$-CD concentration of $6 \%$, no complex is detected with the shorter PEG chains grafted on silica, SiPEG750 (Figure 1b). In addition, a higher rate of complex formation is obtained with free chains of PEG (mPEGMa5K), compared to the same chains grafted on silica nanoparticles (SiPEG5K\#2).

The effect of temperature on the reaction rate was studied at a fixed concentration of $5 \%$ $\alpha-C D$ and four different PEG systems, all containing the same equivalent amount of PEG $(0.3 \%)$. Figure 2 shows the kinetics of complexation with PEG either grafted on the nanoparticles (SiPEG5K, Figure 2a), or free PEG of three different types: with one methoxy end group (mPEG5K, Figure $2 \mathrm{~d}$ ), with one methoxy end and a bulky maleimide group (mPEGMa5K, Figure 2b) or a lower molecular weight (PEG4K, Figure 2c). For all the systems studied, a rise in temperature leads to a decrease in the rate of complexation. Following the model of reaction described by Lo Nostro ${ }^{8,9}$, while diffusion (step 1) is favoured by higher temperatures, the initial threading and sliding of CD are negatively affected by higher temperature, because of the weakening of the interactions responsible for the complexation, such as hydrogen bonds.
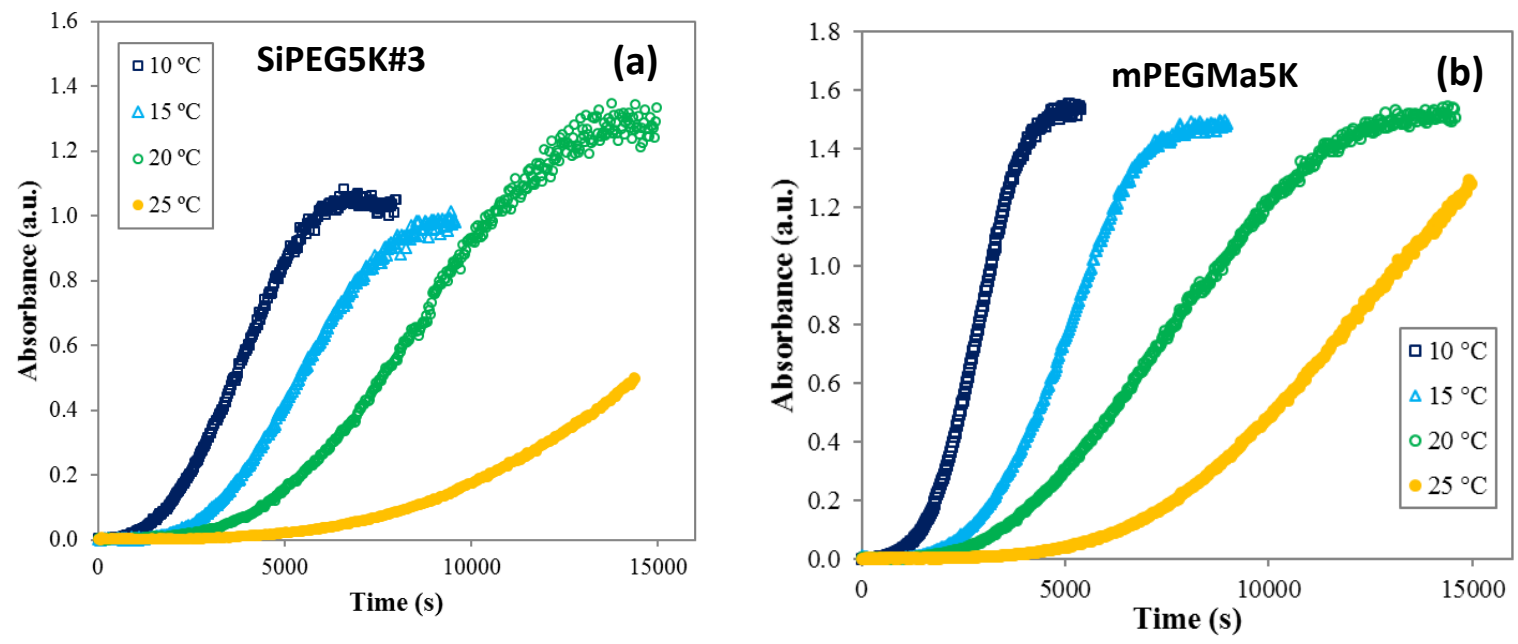

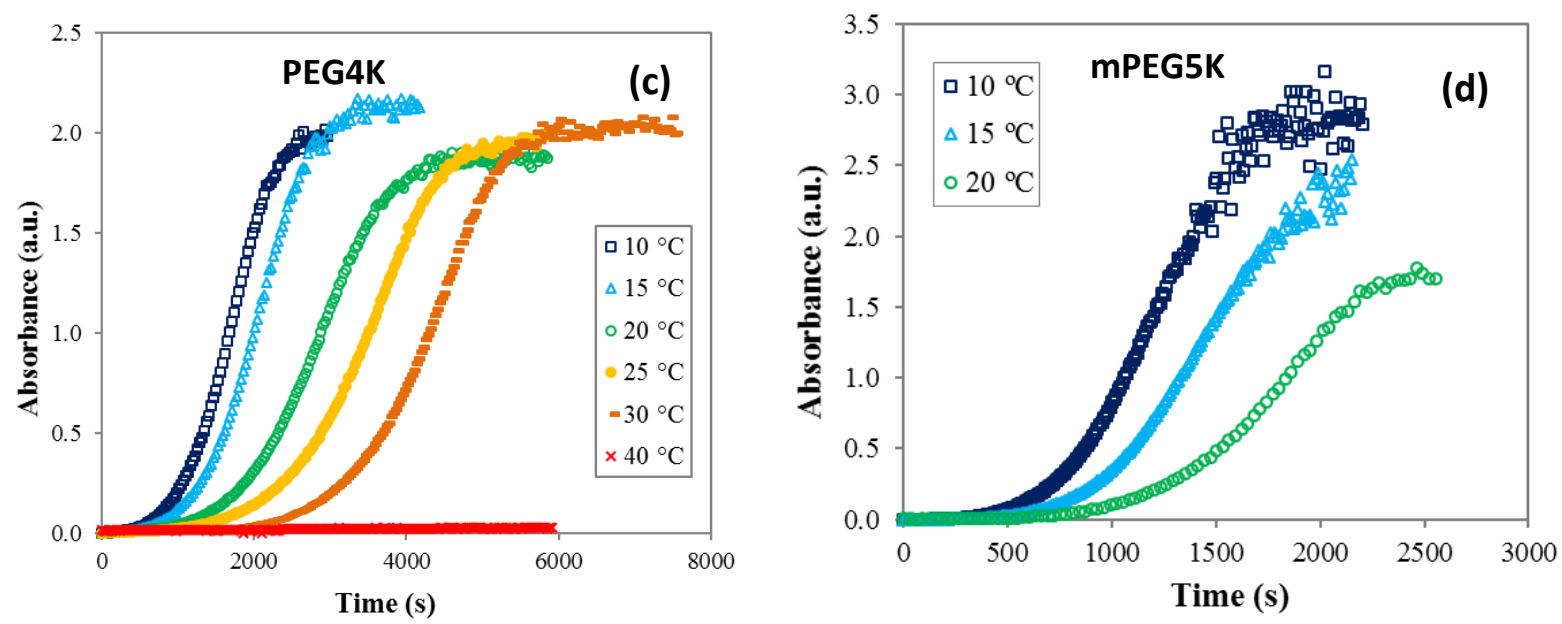

Figure 2. UV-Vis absorbance monitoring the complexation process as a function of temperature in four PEG systems at a constant concentration of 5\% $\alpha$-CD: (a) $0.55 \%$ SiPEG5K\#3 (equivalent 0.3\% PEG); (b) 0.33\% mPEGMa5K; (c) 0.3\%PEG4K; and (d) $0.33 \%$ mPEG5K.

Given the sigmoid shape of the curves, the time corresponding to the inflection point could be used to compare the kinetics of complexation as a function of temperature (Figure S4). These inflection points correspond to the aggregation step, whose onset is located after the threading "delay" time. If the aggregation curves were identical (or very similar) then the inflection points would be a convenient method to compare the curves. The other possibility is to use the onset of the sigmoidal aggregation curves as the threading times (Figure 3). Both approaches give very similar results. Within the four systems studied, two sets of pairs can be distinguished that share a very similar behaviour: (i) on the one hand, SiPEG5K\#3 and mPEGMa5K kinetics are similarly affected by temperature. When the temperature increases, the rate of complexation is strongly modified, and for both systems the inflection point increases, following an exponential trend. Complexation is faster with free PEG (mPEGMa5K) compared to grafted PEG (SiPEG5K\#3). (ii) On the other hand, unmodified free PEG (PEG4K and mPEG5K), while displaying faster complexation kinetics with increasing temperature, are less affected by temperature within this range. Both the 5k molecular weight PEG and the $4 \mathrm{k}$ PEG show extremely similar kinetics. Harada and co-workers ${ }^{2,38}$ determined that the higher the molecular weight of the polymer (above $1000 \mathrm{~g} / \mathrm{mol}$ ), the slower the complexation should be between $\alpha-\mathrm{CD}$ and PEG at equivalent weight concentration. Our experimental results, which show very similar kinetics for both molecular weights, suggest that the end group of the polymers (mPEG5K with a methoxy end and PEG4K with two hydroxyl groups) may impact the kinetics of threading, the less polar methoxy 
end favouring the threading kinetics. This is in agreement with previous studies which have shown that increasing the hydrophobicity of the end group speeds up the complexation rate ${ }^{39}$, which has been proposed as a strategy to separate and purify linear polymers with different terminal groups ${ }^{40}$. Harada et al. have also reported that a higher yield was obtained with methoxy-terminated PEG compared to unmodified PEG ${ }^{38}$.

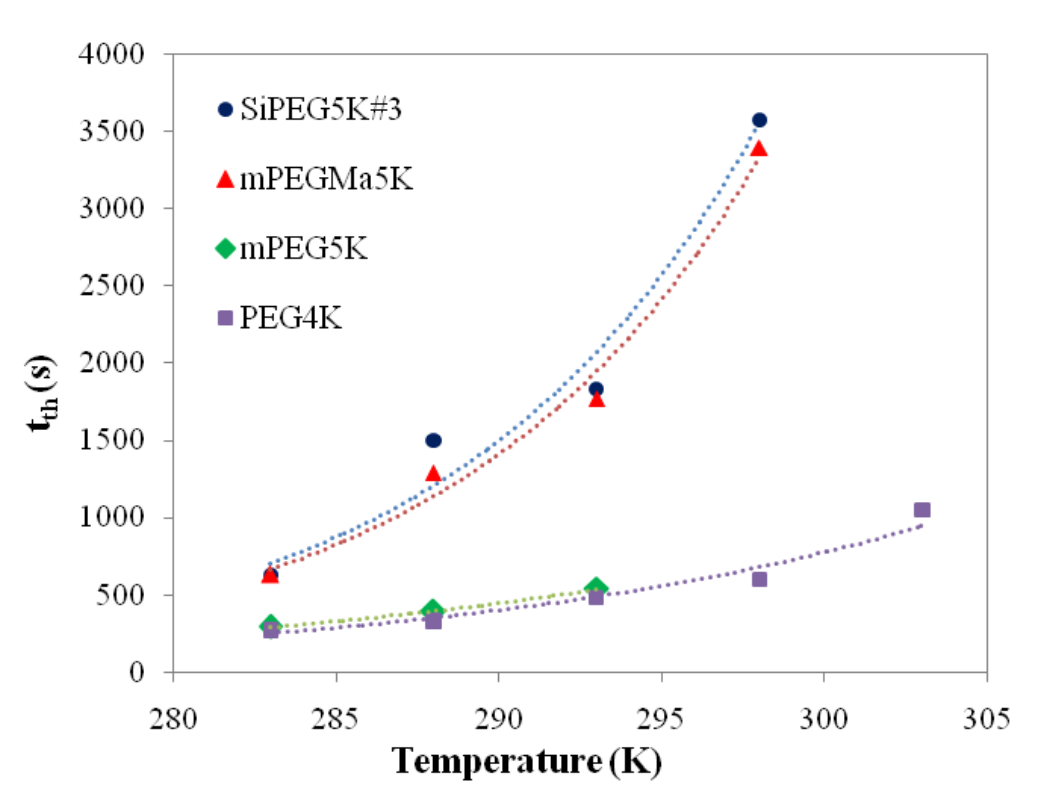

Figure 3. Reaction times at "threading" for the curves obtained from the UV-vis analysis of the complexation between $\alpha-\mathrm{CD}$ and the PEG samples studied as a function of temperature (threading times $t_{t h}$ were considered when absorbances reached a value of 0.01 ).

Figure 3 clearly shows that the kinetics of complexation with the PEGylated nanoparticles are much slower than with the equivalent free polymers. This may be explained both by the presence of only one end accessible for threading of the cyclodextrins, combined to the slower diffusion of the PEG-grafted silica nanoparticles. As mentioned above, maleimide-modified PEG of the same molecular weight (mPEGMa5K) shows similar kinetics to the NP-grafted PEG, suggesting that the maleimide end group presents steric hindrance to the threading process of the $\alpha-\mathrm{CD}$, therefore leaving only one end accessible for complexation (indeed, no reports have been found on the complexation between maleimide and $\alpha-C D$, while complexes are formed with the larger cavity $\beta$ - and $\gamma-C D$ ${ }^{41}$ ). Similarly, PEG carrying bulky substituents on both ends, such as 3,5-dinitrobenzoyl 
or 2,4-dinitrophenyl groups, have been reported to be unable to form a complex with $\alpha$ $\mathrm{CD}^{42}$.

To determine the thermodynamic parameters of the threading, the transition state theory ${ }^{43}$ can be used as a theoretical basis for the modelling ${ }^{44}$ :

$$
\mathrm{PEG}+m(\alpha \mathrm{CD}) \rightleftarrows \sigma^{*} \rightarrow \text { polypseudorotaxane }
$$

Where $\sigma^{*}$ is the formed activated complex (rate step). Within this framework, the threading time $t_{t h}$ depends on the temperature as follows ${ }^{8,9}$ :

$$
\ln \left(T \cdot t_{t h}\right)=-\ln \left(\frac{3 k_{B}}{2 h}\right)-m \cdot \ln [\alpha C D]+\frac{\Delta G_{t h}^{\ddagger}}{R T}
$$

where $m$ is the number of cyclodextrins threaded on each polymer chain, $t_{t h}$ the threading time (measured as the threshold when the turbidity starts to increase, marking the aggregation of the threaded polymers), $[\alpha C D]$ the concentration of cyclodextrin in mol/L, $\Delta G_{t h}^{\ddagger}$ the Gibbs free energy at the threading time, $T$ the temperature and $k_{B}, h$ and $R$, are the Boltzmann constant, the Planck constant and the universal gas constant.

This model was applied to the four systems studied (Figures 2 and 3). For each system, the threading time was determined by assigning the aggregation onset to an absorbance value of 0.01 and resulting $\ln \left(T \cdot t_{t h}\right)$ values were plotted as a function of $1 / T$ (see Figure S5 in Supporting Information). The Gibbs free energy of activation for the threading process $\left(\Delta G_{t h}^{\ddagger}\right)$ and the number of cyclodextrins threaded on each PEG chain $(m)$ were obtained from the slope and the intercept, respectively (Table 2).

Table 2. Values for $m$, the number of cyclodextrins threaded per polymer chain, corresponding molar ratio of $\mathrm{EO}$ and $\alpha-\mathrm{CD}$ units, and $\Delta G_{t h}^{\ddagger}$, the Gibbs energy involved in the formation of the activated complex $\sigma^{*}$.

\begin{tabular}{lccc}
\hline PEG system & $\boldsymbol{m}$ & EO/ $\boldsymbol{\alpha}-\mathbf{C D}$ & $\Delta \boldsymbol{G}_{\boldsymbol{t} \boldsymbol{h}}^{\ddagger}(\mathbf{k J} / \mathbf{m o l})$ \\
\hline SiPEG5K\#3 & 23 & 4.9 & -75.8 \\
mPEGMa5K & 23 & 4.8 & -77.5 \\
mPEG5K & 18 & 6.3 & -44.9 \\
PEG4K & 17 & 5.3 & -41.0 \\
\hline
\end{tabular}


The values of $\Delta G_{t h}^{\ddagger}$ obtained are within the range reported for polymer/CD complexes ${ }^{9}$ and reflect a spontaneous process. These values suggest that the threading process is favoured when PEG is attached to the nanoparticles surface or bears a bulky end group rather than being free in solution, despite the slower kinetics. The number of EO units involved in a complex varies between 5 and 6 , close to the value of $2 \mathrm{EO}$ per $\mathrm{CD}$ for a fully covered chain ${ }^{3,6}$, which may have been reached by using higher concentrations of CD.

In order to check the validity of the energy values obtained, a simpler Arrhenius-like approach was also considered, and the activation enthalpies extrapolated to the onset of the aggregation process (i.e. when the turbidity is extremely low). In this approach, instead of selecting an arbitrary threshold value of the absorbance as the onset of aggregation (such as a value of 0.01 ), a set of absorbances $v s$. time values are required. Considering that the pseudo-first order rate constant of the overall reaction is inversely proportional to time, $\ln \left(1 / t_{a}\right)$ was plotted as a function of $1 / T$ for each constant value of the absorbance $a$. From these van't Hoff-like linear equations, the value of the activation enthalpy was calculated. The results corroborate those obtained following Lo Nostro's model: ${ }^{9}$ the energies at the onset of the aggregation process are ca. $72-74 \mathrm{~kJ} / \mathrm{mol}$ in the case of organosilica nanoparticles and bulky end modified PEG, and are much lower for the free PEG samples, ca. 43-45 kJ/mol (see Supporting Information, Figures S6 and S7). We can also use the turbidity measurements (from Figure 2) to obtain an estimate of the aggregation enthalpies by plotting this time $\ln \left(1 /\left(t_{a}-t_{t h}\right)\right)$ vs. $1 / T$ thereby correcting for the threading step. The shift between the turbidity curves as a function of temperature beyond the threading point, must be related to the enthalpy of aggregation. Thus, the values we obtain from these new van't Hoff plots correspond to the aggregation step (see Supporting Information, Figure S7).

\section{Characterization of the complexes}

Yield of complexation: effect of grafting and polymer length

The complexes obtained after reaction between $\alpha$-CD and SiPEG750, SiPEG5K\#1 or SiPEG5K\#3 were analysed and compared. The mixtures of $\alpha-C D$ solutions with SiPEG750 or SiPEG5K\#1 were turbid at first, and, after three days, a separation into two phases was observed and white precipitates produced. In contrast, samples of SiPEG5K\#3 
with $4.5 \%, 5 \%, 6 \%$ and $7 \% \alpha$-CD formed gels. Figure S8 (Supporting Information) shows the appearance of the solid products (after removal of the supernatant) for $1.3 \%$ SiPEG750, 1.4\% SiPEG5K\#1, and 1.4\% SiPEG5K\#3 with increasing concentrations of $\alpha$-CD. For SiPEG5K\#3, which has a higher grafting density in weight (Table 1), a set of samples at a lower NP concentration $(0.62 \%)$ was also tested to achieve an equivalent PEG concentration, as in the lower PEG-density samples (SiPEG750 and SiPEG5K\#1).

The complexation yields of a range of PEG samples, grafted and free in solution, are shown in Figure 4. Higher yields were obtained for the higher molecular weight grafted PEG, SiPEG5K\#1, compared to SiPEG750 (see also Figure S9 in supporting information). Complexation with SiPEG5K\#3, which has a higher density of PEG grafted on the surface (Table 1), led to even higher complexation yields at both concentrations tested (1.4\% and $0.62 \%)$. The yield was not measurable below $3 \% \alpha-\mathrm{CD}$ for most samples (below 4\% for SiPEG750): below these concentrations, the threading of macrocycles is insufficient to induce the aggregation and bundling of the PPRs.
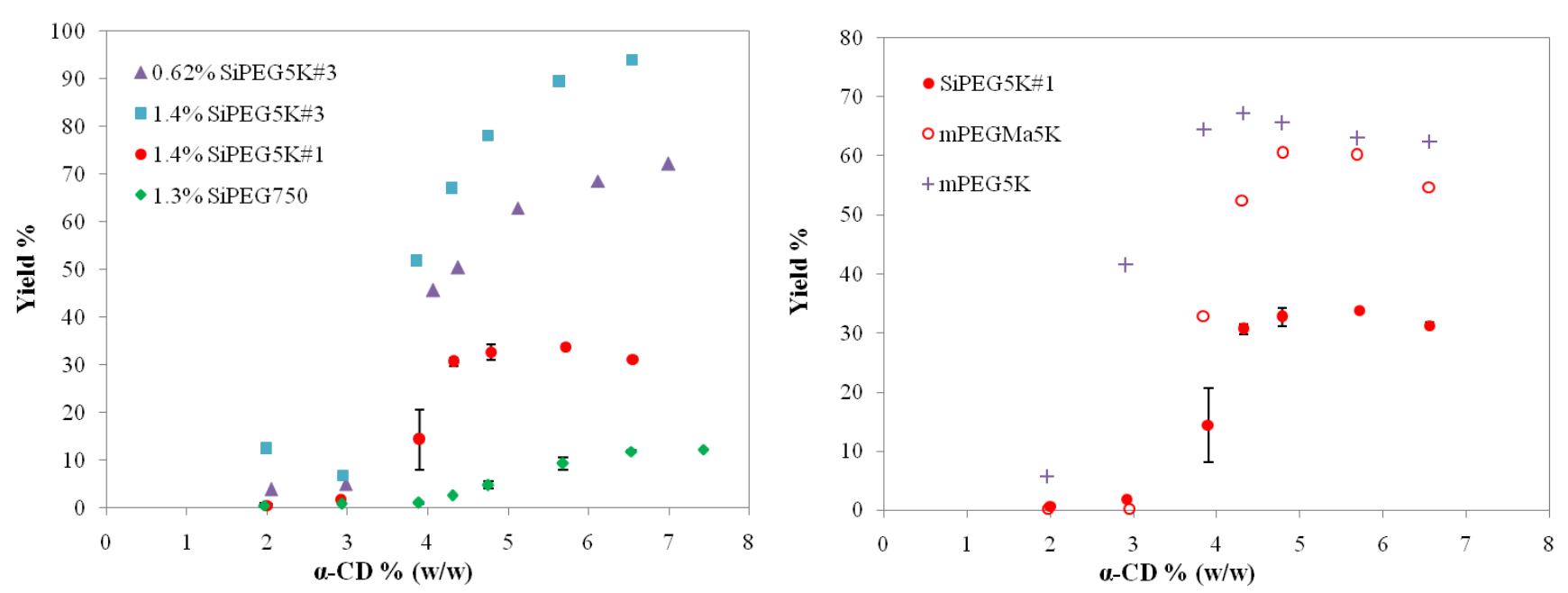

Figure 4.(a) Complexation yields of 1.3\% SiPEG750, 1.4\% SiPEG5K\#1 and 1.4\% SiPEG5K\#3 with varying concentrations of $\alpha$-CD. (b) Complexation yields of $0.33 \%$ mPEG5K, $0.33 \%$ mPEGMa5K and 1.4\% SiPEG5K\#1 (equiv. 0.3\% PEG) with increasing amounts of $\alpha$-CD. Values shown are averages from two experiments.

To assess the effect of grafting on the extent of complexation, experiments were also performed with free polymer in solution (Figure 4b), namely, mPEG5K and mPEGMa5K, the latter bearing a bulky end group not accessible to $\mathrm{CD}$ (potentially a similar effect to grafting one end onto the silica surface). The complexation yields for free mPEG5K and 
mPEGMa5K are higher than for SiPEG5K\#1 nanoparticles with the same concentration of PEG (eq. 0.3\%). Focusing on the free PEG samples, the polymer with the maleimide moiety gave lower complexation yields at low concentrations of $\alpha-\mathrm{CD}$. However, when the concentration of cyclodextrin was increased, both PEGs reached similar yield values. This could indicate that at high concentrations of $\alpha-C D$ the free polymers are saturated and the presence of the bulky maleimide moiety becomes irrelevant. The lower complexation yields for the end-capped PEG obtained at intermediate $\alpha$-CD concentrations should then be attributed to kinetic effects: the gel network structure is hindered when the $\mathrm{CD}$ concentration is low, and the threading process is only occurring through one end. The slight decrease in the yield above a threshold CD concentration suggests the existence of a saturation point for the PEG chains; above this threshold concentration, the weight of the complex remains the same, but the excess of cyclodextrin causes a decrease in the yield value calculated using equations (1) and (2).

In summary, these results show that the following factors affect the yield of complexation negatively: (i) the grafting of PEG on nanoparticles; (ii) shorter chain length; and (iii) lower grafting density. The combination of higher PEG coverage and longer chains led to yields reaching practically $100 \%$. (While it is not excluded that some CD molecules could have remained trapped in the gel without being threaded onto PEG chains, this does suggest a high extent of complexation.)

Figure 5 shows a proposed scheme for the structure of the supramolecular assembly formed between SiPEG NPs and $\alpha$-CD. The aggregation of $\alpha$-CDs threaded on adjacent chains act as physical cross-links, producing a supramolecular network, which is in turn responsible for the formation of a white precipitate or a gel (which are studied in the final section), depending on the concentrations of both components, polymer length and grafting density. When the polymer chains are covalently attached to the surface of nanoparticles, those become embedded into the network. Longer polymer chains and higher grafting density favour complexation and the formation of a more extended network, probably due to the increased local concentration of chain ends through which the macrocycles can thread, and the higher connectivity of a network obtained with longer chains. In a previous study by Sabadini et al. ${ }^{5}$, the PEG chains were physically adsorbed on the surface of silica nanoparticles, rather than chemically bonded. In that scenario also, higher molecular weight PEG induced higher complexation yields. 


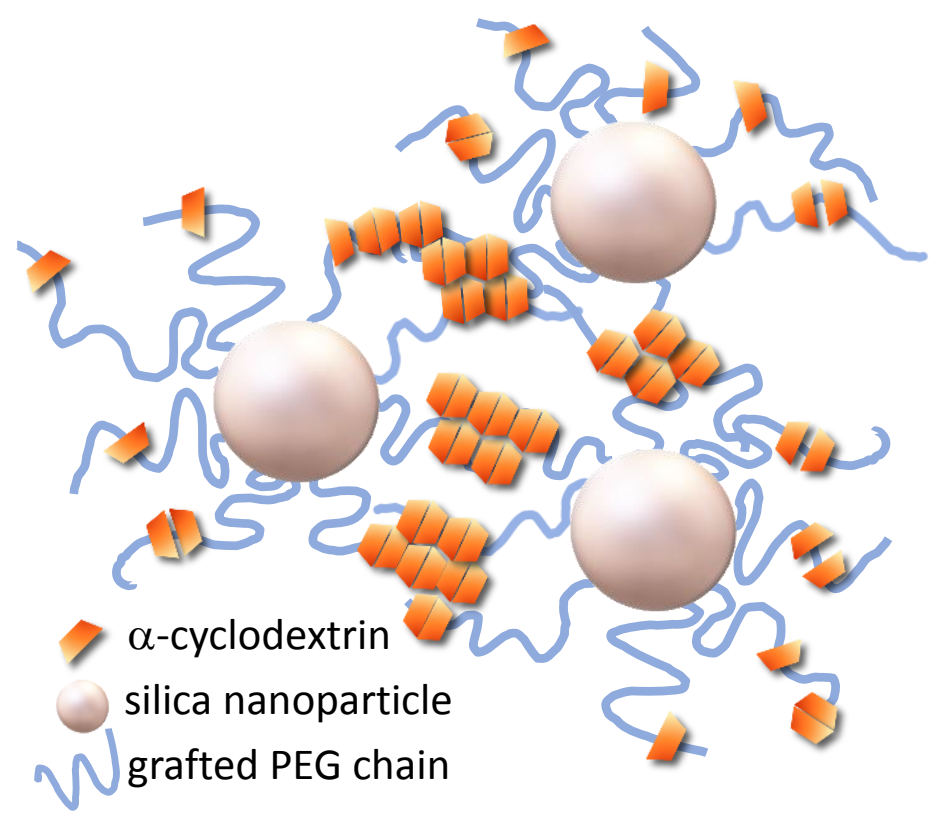

Figure 5. Schematic structure proposed for the supramolecular assembly of SiPEG NPs and $\alpha$ $\mathrm{CD}$, where the threaded $\alpha$-CDs act as physical cross-links causing the self-aggregation of the chains.

Characterization of the complexes by thermogravimetric analysis (TGA): EO/ $\alpha-C D$ ratio Figure 6 shows the TGA curve corresponding to the complex formed (i.e. the solid obtained after the supernatant was removed) between SiPEG5K\#3 and $\alpha-C D$. The curve presents two separate steps between 300 and $400{ }^{\circ} \mathrm{C}$ (which correspond to minima in the derivative curves $\mathrm{dm} / \mathrm{dT}$ at $310^{\circ} \mathrm{C}$ and $388^{\circ} \mathrm{C}$ ). The first step, which is absent in the TGA curves of the thiolated and PEGylated silica, is attributed to the degradation of the cyclodextrin moieties, while the second corresponds to PEG decomposition. Upon complexation, the temperature of degradation of the PEG shifts from $369^{\circ} \mathrm{C}$ to $388^{\circ} \mathrm{C}$, thus demonstrating a certain extent of protection from the threading of cyclodextrins on PEG. From the knowledge of the percentage of PEG in SiPEG5K\#3 (Table 1), the amount of $\alpha-\mathrm{CD}$ in the complex was determined (Table 3), considering that weight loss was due to the decomposition of the organic content (mercaptopropyl groups, PEG and $\alpha-\mathrm{CD}$ ) and that only silica (the inorganic component) remained in the crucible at $1000{ }^{\circ} \mathrm{C}$. 

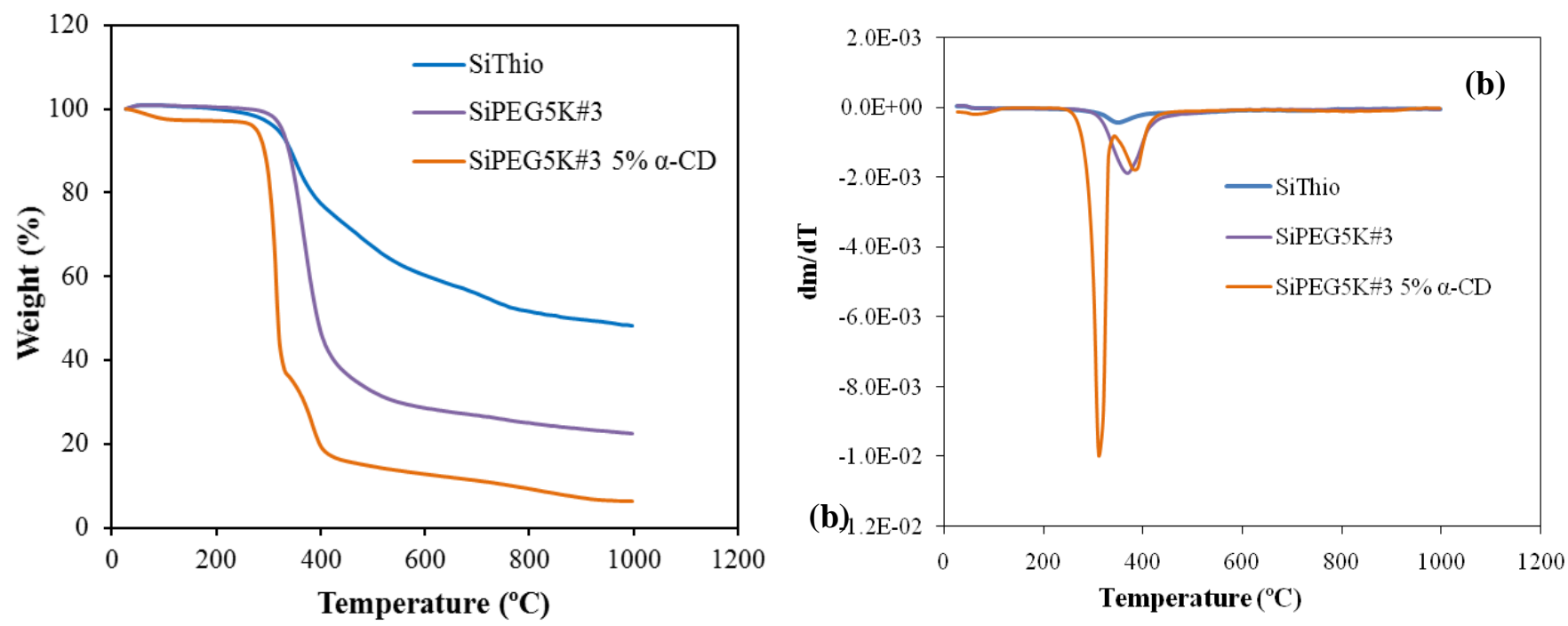

Figure 6. TGA (a) and DTGA (b) data for thiolated silica nanoparticles (SiThio), the PEGylated silica particles SiPEG5K\#3 and the complex formed by $1.4 \%$ SiPEG5K\#3 with $5 \%$ of $\alpha$-CD.

Table 3. Composition of the nanoparticles and their complexes with $\alpha-\mathrm{CD}$ obtained from TGA.

\begin{tabular}{lccccc}
\hline & \multicolumn{5}{c}{ Percentages $(\%$ w/w) } \\
\cline { 2 - 6 } Substance & $\mathrm{SiO}_{2}$ & $\mathrm{R}-\mathrm{SH}$ & PEG5K & $\alpha-\mathrm{CD}$ & $\mathrm{H}_{2} \mathrm{O}$ \\
\hline SiThio & 48 & 52 & - & - & - \\
SiPEG5K\#3 & 23 & 24 & 53 & - & - \\
$\begin{array}{l}\text { SiPEG5K\#3 } \\
+5 \% \boldsymbol{\alpha}-\mathbf{C D}\end{array}$ & 6 & 7 & 15 & $\mathbf{6 9}$ & 3 \\
\hline
\end{tabular}

The compositions of PEG and $\alpha-\mathrm{CD}$ from Table 3 were used to establish the EO: $\alpha-\mathrm{CD}$ mole ratio in the complex as 5:1 (Supporting Information, section 3), in agreement with the value obtained from thermodynamic analysis (Table 2). These calculations were performed assuming that all the $\alpha-\mathrm{CD}$ was part of the pseudopolyrotaxane; however, some unthreaded macrocycles could have been trapped in the supramolecular aggregates. The maximum possible ratio in these polyrotaxane structures is $2 \mathrm{EO}$ units per $\alpha-\mathrm{CD}^{3,6}$, suggesting that there is still scope for more extensive threading. It is possible however that, with chains grafted on nanoparticles, threading close to the silica surfaces becomes hindered at high surface coverages due to steric hindrance. TGA experiments with other 
PEGylated NPs (batch \#1 and \#3) and varying $\alpha-C D$ concentrations all show a similar behaviour (Supporting Information, Figure S10).

\section{Characterization of the complexes by FTIR spectroscopy}

The surface functionalization of nanoparticles can be efficiently monitored using infrared spectroscopy. The absorption from functional groups present on their surface can be easily identified using this technique. FTIR-ATR spectra of $\alpha$-CD, mPEGMa5K, SiPEG5K\#3 and the complex formed by SiPEG5K\#3 with 5\% $\alpha-\mathrm{CD}$ are shown in Figure 7 (an expansion of the fingerprint region can be found in Figure S11, Supporting Information). As can be seen, the spectra of mPEGMa5K and SiPEG5K\#3 are very similar, with the exception of the additional band at $1026 \mathrm{~cm}^{-1}$ in SiPEG5K\#3, which corresponds to the $\mathrm{Si}-\mathrm{OH}$ or $\mathrm{Si}-\mathrm{OR}$ stretching vibration ${ }^{45}$.

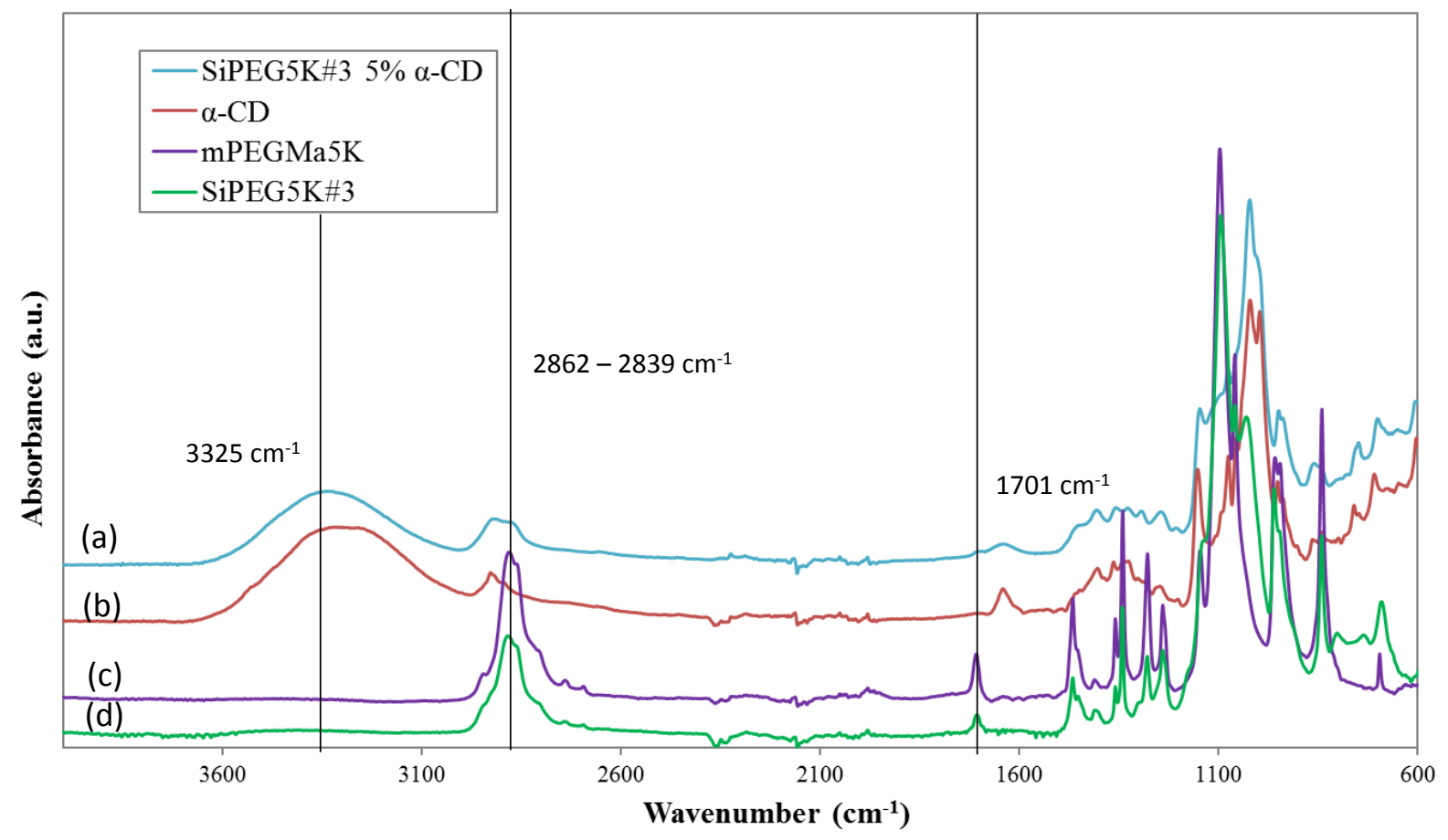

Figure 7. FTIR spectra of $\alpha$-CD (b), mPEGMa5K (c), SiPEG5K\#3 (d) and SiPEG5K\#3 with 5\% $\alpha-\mathrm{CD}(\mathrm{a})$.

The $\alpha$-CD spectrum presents a broad band at $3273 \mathrm{~cm}^{-1}$, corresponding to the $\mathrm{O}-\mathrm{H}$ stretching, which is shifted to higher frequency $\left(3325 \mathrm{~cm}^{-1}\right)$ in the complex, probably due to hydrogen bonding between the cyclodextrin $\mathrm{O}-\mathrm{H}$ groups and the ether oxygen atoms in the threaded PEG ${ }^{46}$. The bands corresponding to C-H stretching modes appear at 2904 
$\mathrm{cm}^{-1}$ for $\alpha-C D$ and at lower frequency $\left(2839-2862 \mathrm{~cm}^{-1}\right)$ for the SiPEG5K\#3 nanoparticles. In the spectrum of the complex, both bands appear overlapped. At 1701 $\mathrm{cm}^{-1}$, a band corresponding to $\mathrm{C}=\mathrm{O}$ stretching from the maleimide moiety ${ }^{47}$ is present in both the SiPEG5K\#3 and mPEGMa5K spectra, so the small band in the complex at this same wavenumber could be associated to this stretching mode (which is absent in the $\alpha$ CD spectrum). The most intense band in SiPEG5K\#3 at $1095 \mathrm{~cm}^{-1}$ corresponds to the C$\mathrm{O}$ stretching from the polymer chain ${ }^{48}$. However, this band cannot be detected in the complex spectrum. In contrast, the characteristic vibration mode of $\alpha-\mathrm{CD}$ at $1020 \mathrm{~cm}^{-1}$ (C-O stretching) clearly appears in the complex spectrum. Overall, the vibration modes of $\alpha-\mathrm{CD}$ are predominant in the complex spectrum, which agrees with the high CD/PEG ratio found in the TGA experiments.

\section{Phase behaviour and rheological properties of the hybrid gels}

At relatively high CD and PEG concentrations, the complexation of SiPEG5K\#3 with $\alpha-$ $\mathrm{CD}$ induces the formation of gels, as has been reported for free PEG in solution, or other copolymers ${ }^{5-7,18,19,49}$. These gels are formed by the aggregation of the inclusion complexes into microcrystalline regions, which act as physical crosslinks for the networks. They are receiving increasing attention as biomaterials, mostly because of their injectability and self-healing properties ${ }^{18,19}$. The gels reported here present the additional attractive feature of embedding silica nanoparticles within their network. Nanoparticles with lower grafting density (SiPEG5K\#1) or lower molecular weight PEG (SiPEG750) did not form gels over the range of $\alpha$-CD concentrations studied, showing the importance of both polymer length and surface coverage in producing self-supported gels.

The samples prepared by combining different concentrations of $\alpha-C D$ and nanoparticles were visualised and classified according to their aspect (Figure 8): solutions (transparent, no traces of precipitate); biphasic systems (turbid at first and separating in two phases when left to stand); weak gels (supporting their own weight when inverted but easily broken when shaken); and gels. Weak gels were obtained for concentrations above $0.2 \%$ SiPEG5K\#3 and a minimum of $4.5 \% \alpha-\mathrm{CD}$, corresponding to a ratio of $4 \mathrm{EO}$ for $1 \mathrm{CD}$ (or $0.25 \mathrm{CD} / \mathrm{EO}$ molar ratio). 

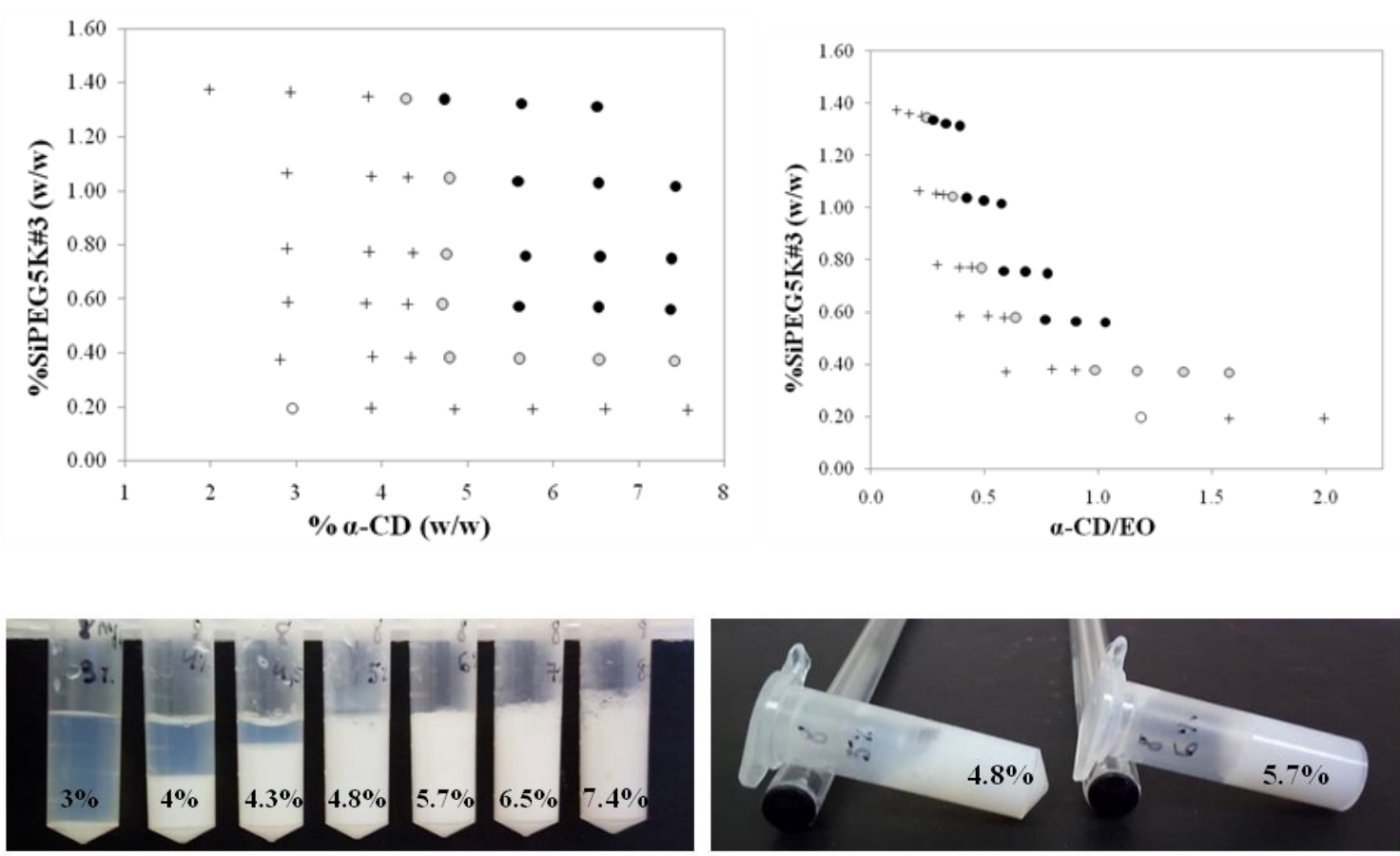

Figure 8. Phase diagram for mixtures of SiPE5K\#3 nanoparticles and $\alpha$-CD in aqueous

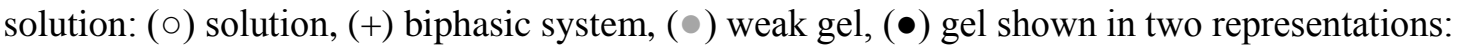
as a function of $\alpha-\mathrm{CD}$ wt.\% (top left) and $\alpha-\mathrm{CD} / \mathrm{EO}$ molar ratio (top right). Bottom: Pictures showing the appearance of complexes of SiPEG5K\#3 $(0.8 \% \mathrm{w} / \mathrm{w})$ with $\alpha$-CD up to $7.4 \% \mathrm{w} / \mathrm{w}$ after 7 days (left); samples with 4.8\% (weak gel) and 5.7\% (gel) $\alpha$-CD after 7 days (right).

In addition, mixtures of mPEGMa5K with $\alpha$-CD at a fixed PEG concentration $(0.3 \%)$ were produced to compare the results of the end-capped PEG with those of the PEGylated nanoparticles. For low cyclodextrin concentrations (2-3\%), neither turbidity nor precipitation were observed. Samples with $4-5 \% \alpha-C D$ yielded two phases. Increasing cyclodextrin concentration further (between $6 \%$ and 10\%) led to the formation of thick gels.

Oscillatory rheology was performed on the supramolecular gels resulting from the complexation of $\alpha$-CD with either grafted PEG (SiPEG5K\#2) (Figure 9a), or free endcapped PEG (mPEGMa5K) (Figure 9b), with concentrations ranging between $6 \%$ and $12 \%$. The elastic modulus $\left(G^{\prime}\right)$ of the nanocomposite gels (Figure 9a) is found to be highly independent of frequency over the range measured, reflecting a highly structured, predominantly solid-like material, reminiscent of chemically crosslinked gels. The reproducibility of the measurements was relatively poor, and improved with the amount of $\mathrm{CD}$ added. The limited reproducibility is not surprising, given the nature of these gels 
which are formed through the diffusion-controlled threading of CDs on the chains, and their subsequent aggregation (akin to a phase separation process). This random process is thus expected to lead to quite some variability in gel structure and thus in their rheological properties, as previously observed in the diffusion-controlled crosslinking of gels obtained by an enzymatic process ${ }^{50}$. The precise preparation protocol (e.g. order of mixing, concentration of stock solutions used, and stirring) was also seen to impact the final rheological behaviour, in agreement with different formation pathways leading to slightly different structures. The rheology data show a slight weakening of the gels with increasing amount of cyclodextrins; this suggests that, once a threshold concentration of cyclodextrins has been reached that provides connectivity to the network (cf phase diagrams, Figure 8), additional cyclodextrins do not create additional elastically active junctions and may even be detrimental to the rheological properties.
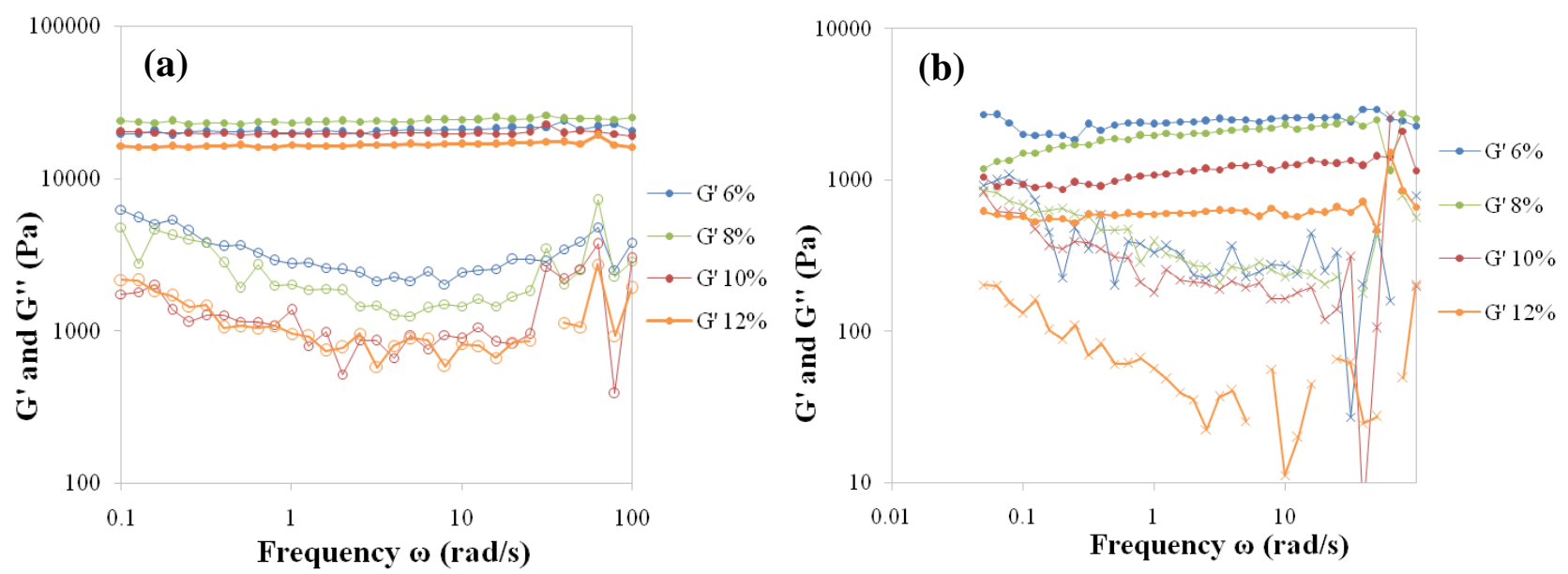

Figure 9. (a) Dynamic frequency sweep measurements for 0.5\% SiPEG5K\#2 and SiPEG5K\#3 ( $0.3 \%$ eq PEG) with increasing weight $\%$ of $\alpha-\mathrm{CD}$. The storage modulus $G$ ' is shown by filled symbols and the loss modulus $G$ "' by empty symbols. (b) Dynamic frequency sweeps for free, end-capped PEG (mPEGMa5K, 0.3\%) with increasing weight $\%$ of $\alpha$-CD. (Data are averages corresponding to two or three measurements. Two set of samples were prepared with batch \#2 and one set with batch \#3.)

The gels obtained from the threading of free PEG (Figure 9b) reveal lower values of $G$ ' than the gels obtained with silica-grafted PEG, reflecting weaker, more yielding gels. The incorporation of inorganic silica within the connected network thus improves the elastic 
properties of the gels by one order of magnitude (Figure S12); this reinforcement with nanoparticles has often been observed with nanocomposite gels ${ }^{21,22}$.

\section{CONCLUSIONS}

The complexation and formation of supramolecular assemblies between $\alpha$-cyclodextrin $(\alpha-C D)$ and PEGylated organosilica nanoparticles was studied. Nanoparticles with PEG of different molecular weights (750 and $5000 \mathrm{~g} / \mathrm{mol}$ ) and different grafting density were used, as well as three different free polymers: PEG4K, mPEG5K, and mPEGMa5K, the latter bearing an end modified with a maleimide moiety.

Nanoparticles with a higher coverage of PEG and longer polymer chains (5k vs. 750) showed faster kinetics of complexation and higher complexation yields at equilibrium, indicating that both the length of the polymer and the grafting density are key parameters that dictate the speed of threading and the final supramolecular structures. For all the systems investigated, the complexation rate decreased upon increasing the temperature, although the free unmodified PEGs were less affected by temperature, and mPEGMa5K (PEG with a bulky maleimide endgroup) showed kinetics of complexation quite similar to the nanoparticles, as they present also one chain end through which CDs cannot enter. Gravimetric experiments showed that the complexation yields were lower in the presence of nanoparticles than with free polymer. Both thermodynamic analysis, using transition state theory, and thermogravimetry suggested a complexation ratio of 1 macrocycle for 5-6 EO units, a comparable but lower coverage to the maximum of 1CD:2EO ratio reported by $\operatorname{Harada}^{3,38}$.

Above a threshold concentration of $\alpha-\mathrm{CD}$ and PEG, either grafted to nanoparticles or free in solution, self-supporting gels were formed. Nanoparticles bearing a lower grafting density of PEG, or with the lower molecular weight PEG, did not form gels. Oscillatory rheology measurements performed on the gels showed a highly viscoelastic, predominantly solid-like behaviour, with the elastic modulus $\left(G^{\prime}\right)$ broadly independent of frequency. The networks with embedded silica nanoparticles presented superior elastic properties compared to the equivalent free polymer gel samples, suggesting that the presence of the nanoparticles reinforced the gels.

Overall, our results elucidate the key parameters that direct the kinetics of complexation and the final equilibrium structures for a new type of soft hybrid materials based on hostguest interactions. In the current context of the search for novel hydrogels for biomedical 
applications, either for delivery applications or tissue engineering, this work provides a facile route towards supramolecular gels comprising soft nanoparticle gels, combining organic and inorganic materials.

Supporting Information. S1: Structures of molecules. S2: TGA data on the organosilica nanoparticles. S3: DLS data on the nanoparticles. S4: a. example of absorbance vs. time curve, b. Reaction times at the inflection points $t_{i}$ of the curves obtained from UV-vis analysis as a function of temperature (corresponding to Figure 3$) . S 5: \ln \left(T \cdot t_{t h}\right)$ as a function of 1/T for the four systems studied. S6: van't Hoff-like plots for mPEGMa5K and $\alpha \mathrm{CD}$. S7: Enthalpy plots from turbidity experiments as a function of the absorbance. S8: pictures showing the appearance of the complexes and gels. S9: expansion of Figure 4. S10: TGA data on the complexes (and calculations to determine EO:CD ratios from the plots). S11: FTIR spectra from the complexes. S12: Values of the elastic moduli at 1 $\mathrm{rad} / \mathrm{s}$ for the gels with and without silica. Table S1: threading times from turbidity curves.

\section{ACKNOWLEDGEMENTS}

Financial support from project MAT2014-59116-C2-2-R of the Spanish MINECO and the University of Navarra's School of Sciences for the internship grant of M.S.-G. is acknowledged. D.B.K. gratefully acknowledges the British Council Newton-Al-Farabi Partnership Programme, the Researcher Links Post-doctoral Mobility Grant (216046068) for financial support of his postdoctoral fellowship at the University of Reading.

\section{REFERENCES}

(1) Crini, G. Review: A History of Cyclodextrins. Chem. Rev. 2014, 114 (21), 10940-10975.

(2) Harada, A.; Kamachi, M. Complex Formation between Poly(Ethylene Glycol) and $\alpha$-Cyclodextrin. Macromolecules 1990, 23 (10), 2821-2823.

(3) Li, J.; Harada, A.; Kamachi, M. Sol-Gel Transition during Inclusion Complex Formation between $\alpha$-Cyclodextrin and High Molecular Weight Poly(Ethylene Glycol)s in Aqueous Solution. Polymer Journal. 1994, pp 1019-1026.

(4) Wenz, G.; Han, B. H.; Müller, A. Cyclodextrin Rotaxanes and Polyrotaxanes. 
Chem. Rev. 2006, 106 (3), 782-817.

(5) Sabadini, E.; Cosgrove, T.; Taweepreda, W. Complexation between $\alpha$ Cyclodextrin and Poly(Ethylene Oxide) Physically Adsorbed on the Surface of Colloidal Silica. Langmuir 2003, 19 (11), 4812-4816.

(6) Li, J.; Ni, X.; Leong, K. W. Injectable Drug-Delivery Systems Based on Supramolecular Hydrogels Formed by Poly(Ethylene Oxide)s and $\alpha$ Cyclodextrin. J. Biomed. Mater. Res. 2003, 65A (2), 196-202.

(7) Dreiss, C. A.; Cosgrove, T.; Newby, F. N.; Sabadini, E. Formation of a Supramolecular Gel between $\alpha$-Cyclodextrin and Free and Adsorbed PEO on the Surface of Colloidal Silica: Effect of Temperature, Solvent, and Particle Size. Langmuir 2004, 20 (21), 9124-9129.

(8) Ceccato, M.; Nostro, P. Lo; Baglioni, P. Cyclodextrin / Polyethylene Glycol Polyrotaxane : A Study of the Threading Process. Langmuir 1997, 7463 (96), 2436-2439.

(9) Lo Nostro, P.; Giustini, L.; Fratini, E.; Ninham, B. W.; Ridi, F.; Baglioni, P. Threading, Growth, and Aggregation of Pseudopolyrotaxanes. J. Phys. Chem. B 2008, 112 (4), 1071-1081.

(10) Appel, E. A.; del Barrio, J.; Loh, X. J.; Scherman, O. A. Supramolecular Polymeric Hydrogels. Chem. Soc. Rev. 2012, 41 (18), 6195-6214.

(11) Simões, S. M. N.; Rey-Rico, A.; Concheiro, A.; Alvarez-Lorenzo, C. Supramolecular Cyclodextrin-Based Drug Nanocarriers. Chem. Commun. 2015, 51 (29), 6275-6289.

(12) Manakker, F. Van De; Vermonden, T.; Nostrum, C. F. Van; Hennink, W. E. Cyclodextrin-Based Polymeric Materials: Synthesi , Properties, and Pharmaceutical/Biomedical Applications. Biomacromolecules 2009, 10 (12), 3157-3175.

(13) Yuen, F.; Tam, K. C. Cyclodextrin-Assisted Assembly of Stimuli-Responsive Polymers in Aqueous Media. Soft Matter 2010, 6 (19), 4613-4630.

(14) Loebel, C.; Rodell, C. B.; Chen, M. H.; Burdick, J. A. Shear-Thinning and SelfHealing Hydrogels as Injectable Therapeutics and for 3D-Printing. Nat. Protoc. 2017, 12 (8), 1521-1541.

(15) Sun, T.; Zhang, Y. S.; Pang, B.; Hyun, D. C.; Yang, M.; Xia, Y. Engineered Nanoparticles for Drug Delivery in Cancer Therapy. Angew. Chemie - Int. Ed. 2014, 53 (46), 12320-12364.

(16) Gonzalez-Gaitano, G.; Isasi, J.; Velaz, I.; Zornoza, A. Drug Carrier Systems Based on Cyclodextrin Supramolecular Assemblies and Polymers: Present and Perspectives. Curr. Pharm. Des. 2016, 23 (999), 411-432.

(17) Singh, A.; Zhan, J.; Ye, Z.; Elisseeff, J. H. Modular Multifunctional Poly(Ethylene Glycol) Hydrogels for Stem Cell Differentiation. Adv. Funct. Mater. 2013, 23 (5), 575-582. 
(18) Li, J.; Li, X.; Ni, X.; Wang, X.; Li, H.; Leong, K. W. Self-Assembled Supramolecular Hydrogels Formed by Biodegradable PEO-PHB-PEO Triblock Copolymers and $\alpha$-Cyclodextrin for Controlled Drug Delivery. Biomaterials 2006, 27 (22), 4132-4140.

(19) Li, J.; Loh, X. J. Cyclodextrin-Based Supramolecular Architectures: Syntheses, Structures, and Applications for Drug and Gene Delivery. Adv. Drug Deliv. Rev. 2008, 60 (9), 1000-1017.

(20) Erickson, R. P.; Fiorenza, M. T. A Hopeful Therapy for Niemann-Pick C Diseases. Lancet 2017, 390 (10104), 1720-1721.

(21) Da Silva, M. A.; Dreiss, C. A. Soft Nanocomposites: Nanoparticles to Tune Gel Properties. Polym. Int. 2016, 65 (3), 268-279.

(22) Serra-Gómez, R.; Dreiss, C. A.; González-Benito, J.; González-Gaitano, G. Structure and Rheology of Poloxamine T1107 and Its Nanocomposite Hydrogels with Cyclodextrin-Modified Barium Titanate Nanoparticles. Langmuir 2016, 32 (25), 6398-6408.

(23) Niu, Y.; Guo, T.; Yuan, X.; Zhao, Y.; Ren, L. An Injectable Supramolecular Hydrogel Hybridized with Silver Nanoparticles for Antibacterial Application. Soft Matter 2018, 14 (7), 1227-1234.

(24) Joseph, J.; Dreiss, C. A.; Cosgrove, T.; Dreiss, A. Stretching a Polymer Brush by Making in Situ Cyclodextrin Inclusion Complexes Stretching a Polymer Brush by Making in Situ Cyclodextrin Inclusion Complexes. Langmuir 2008, 24 (18), 10005-10010.

(25) Takahashi, S.; Yamada, N. L.; Ito, K.; Yokoyama, H. Inclusion Complex of $\alpha$ Cyclodextrin with Poly(Ethylene Glycol) Brush. Macromolecules 2016, 49 (18), 6947-6952.

(26) Coelho, J. P.; Gonz??lez-Rubio, G.; Delices, A.; Barcina, J. O.; Salgado, C.; ??vila, D.; Pe??a-Rodr??guez, O.; Tardajos, G.; Guerrero-Mart??nez, A. Polyrotaxane-Mediated Self-Assembly of Gold Nanospheres into Fully Reversible Supercrystals. Angew. Chemie - Int. Ed. 2014, 53 (47), 12751-12755.

(27) Paulo Coelho, J.; Osío Barcina, J.; Junquera, E.; Aicart, E.; Tardajos, G.; GómezGraña, S.; Cruz-Gil, P.; Salgado, C.; Díaz-Núñez, P.; Peña-Rodríguez, O.; et al. Supramolecular Control over the Interparticle Distance in Gold Nanoparticle Arrays by Cyclodextrin Polyrotaxanes. Nanomaterials 2018, 8 (3), 168.

(28) Yu, J.; Ha, W.; Sun, J. N.; Shi, Y. P. Supramolecular Hybrid Hydrogel Based on Host-Guest Interaction and Its Application in Drug Delivery. ACS Appl. Mater. Interfaces 2014, 6 (22), 19544-19551.

(29) Watermann, A. Mesoporous Silica Nanoparticles as Drug Delivery Vehicles in Cancer. Nanomaterials 2017, 7 (7), 189.

(30) Wang, Y.; Zhao, Q.; Han, N.; Bai, L.; Li, J.; Liu, J.; Che, E.; Hu, L.; Zhang, Q.; Jiang, T.; et al. Mesoporous Silica Nanoparticles in Drug Delivery and Biomedical Applications. Nanomedicine Nanotechnology, Biol. Med. 2015, 11 (2), 313-327. 
(31) Irmukhametova, G. S.; Mun, G. A.; Khutoryanskiy, V. V. Thiolated Mucoadhesive and PEGylated Nonmucoadhesive Organosilica Nanoparticles from 3-Mercaptopropyltrimethoxysilane. Langmuir 2011, 27 (15), 9551-9556.

(32) Al Mahrooqi, J. H.; Mun, E. A.; Williams, A. C.; Khutoryanskiy, V. V. Controlling the Size of Thiolated Organosilica Nanoparticles. Langmuir 2018, acs.langmuir.8b01556.

(33) Mun, E. A.; Williams, A. C.; Khutoryanskiy, V. V. Adhesion of Thiolated Silica Nanoparticles to Urinary Bladder Mucosa: Effects of PEGylation, Thiol Content and Particle Size. Int. J. Pharm. 2016, 512 (1), 32-38.

(34) Irmukhametova, G. S.; Fraser, B. J.; Keddie, J. L.; Mun, G. A.; Khutoryanskiy, V. V. Hydrogen-Bonding-Driven Self-Assembly of PEGylated Organosilica Nanoparticles with Poly(Acrylic Acid) in Aqueous Solutions and in Layer-byLayer Deposition at Solid Surfaces. Langmuir 2012, 28 (1), 299-306.

(35) Mun, E. A.; Hannell, C.; Rogers, S. E.; Hole, P.; Williams, A. C.; Khutoryanskiy, V. V. On the Role of Specific Interactions in the Diffusion of Nanoparticles in Aqueous Polymer Solutions. Langmuir 2014, 30 (1), 308-317.

(36) Mun, E. A.; Morrison, P. W. J.; Williams, A. C.; Khutoryanskiy, V. V. On the Barrier Properties of the Cornea: A Microscopy Study of the Penetration of Fluorescently Labeled Nanoparticles, Polymers, and Sodium Fluorescein. Mol. Pharm. 2014, 11 (10), 3556-3564.

(37) Riddick, T. M. Control of Colloid Stability through Zeta Potential.; Livingston: Wynnewood, Pa., 1968.

(38) Harada, A.; Li, J.; Kamachi, M. Preparation and Properties of Inclusion Complexes of Poly(Ethylene Glycol) with $\alpha$-Cyclodextrin. Macromolecules 1993, 26 (21), 5698-5703.

(39) Xue, J.; Chen, L.; Zhou, L.; Jia, Z.; Wang, Y.; Zhu, X.; Yan, D. Effect of End Groups on Complexation Kinetics between Cyclodextrins and Guest Polymers. $J$. Polym. Sci. Part B Polym. Phys. 2006, 44 (April), 2050-2057.

(40) Xue, J.; Zhou, L.; He, P.; Zhu, X.; Yan, D.; Jiang, X. Supramolecular End-Group Separation of Linear Polymers with Different Terminals through Host-Guest Interaction. J. Incl. Phenom. Macrocycl. Chem. 2008, 61 (1-2), 83-88.

(41) Kawamura, M.; Higashi, M. Induced Circular Dichroism and Magnetic Circular Dichroism Spectra of Maleimide and Related Molecules. Helv. Chim. Acta 2003, 86 (7), 2342-2348.

(42) Harada, A.; Li, J.; Kamachi, M. Macromolecular Recognition: Formation of Inclusion Complexes of Polymers with Cyclodextrins. Proc. Japan Acad. Ser. B Phys. Biol. Sci. 1993, 69 (3), 39-44.

(43) Laidler, K. J. Chemical Kinetics, Third.; Prentice Hall: New Jersey, 1987.

(44) Lo Nostro, P.; Lopes, J. R.; Cardelli, C. Formation of Cyclodextrin-Based Polypseudorotaxanes: Solvent Effect and Kinetic Study. Langmuir 2001, 17 (15), 4610-4615. 
(45) Chakraborty, S.; Biswas, S.; Sa, B.; Das, S.; Dey, R. In Vitro \&amp; in Vivo Correlation of Release Behavior of Andrographolide from Silica and PEG Assisted Silica Gel Matrix. Colloids Surfaces A Physicochem. Eng. Asp. 2014, 455 (Complete), 111-121.

(46) Rusa, C. C.; Tonelli, A. E. Separation of Polymers by Molecular Weight through Inclusion Compound Formation with Urea and $\alpha$-Cyclodextrin Hosts.

Macromolecules 2000, 33 (5), 1813-1818.

(47) Altintas, Z.; Karatas, S.; Kayaman-Apohan, N.; Gungor, A. The Maleimide Modified Epoxy Resins for the Preparation of UV-Curable Hybrid Coatings. Polym. Adv. Technol. 2011, 22 (2), 270-278.

(48) Semsarzadeh, M. A. L. I.; Amiri, S. Preparation and Properties of Polyrotaxane from $\alpha$-Cyclodextrin and Poly ( Ethylene Glycol ) with Poly ( Vinyl Alcohol ). Bull. Mater. Sci. 2013, 36 (6), 989-996.

(49) Simões, S. M. N.; Veiga, F.; Torres-Labandeira, J. J.; Ribeiro, A. C. F.; SandezMacho, M. I.; Concheiro, A.; Alvarez-Lorenzo, C. Syringeable Pluronic- $\alpha-$ Cyclodextrin Supramolecular Gels for Sustained Delivery of Vancomycin. Eur. J. Pharm. Biopharm. 2012, 80 (1), 103-112.

(50) Bode, F.; Da Silva, M. A.; Drake, A. F.; Ross-Murphy, S. B.; Dreiss, C. A. Enzymatically Cross-Linked Tilapia Gelatin Hydrogels: Physical, Chemical, and Hybrid Networks. Biomacromolecules 2011, 12 (10), 3741-3752. 


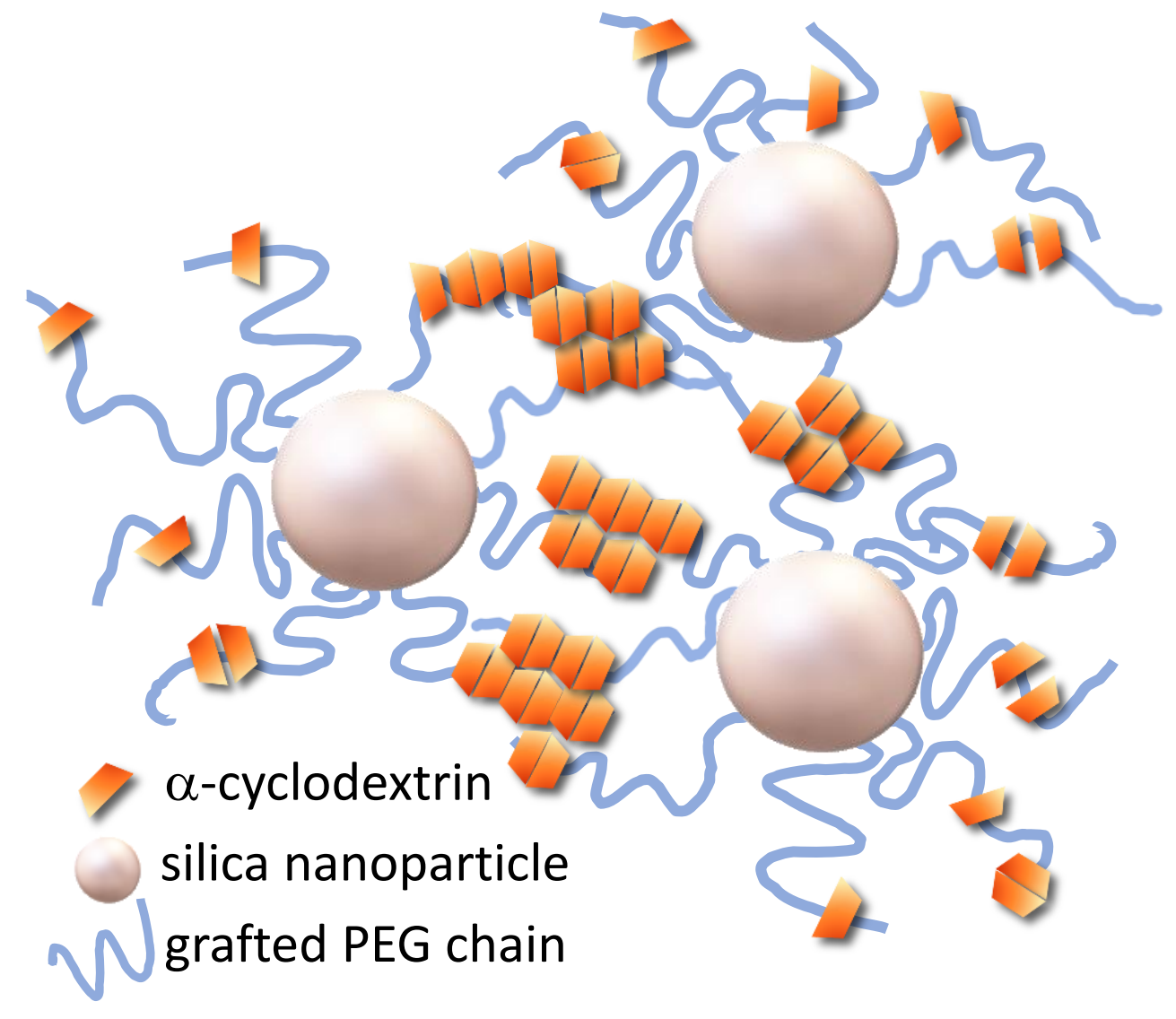

